\title{
Combined alkylation and histone deacetylase inhibition with EDO-S101 has significant therapeutic activity against brain tumors in preclinical models
}

\author{
Yushi Qiu ${ }^{1}$, Zhimin Li ${ }^{1}$, John A. Copland ${ }^{1}$, Thomas Mehrling ${ }^{3}$ and Han W. Tun ${ }^{1,2}$ \\ ${ }^{1}$ Department of Cancer Biology, Mayo Clinic, Jacksonville, FL 32224, USA \\ ${ }^{2}$ Division of Hematology and Medical Oncology, Mayo Clinic, Jacksonville, FL 32224, USA \\ ${ }^{3}$ Mundipharma-EDO GmbH, 4052 Basel, Switzerland \\ Correspondence to: Han W. Tun, email:Tun.Han@mayo.edu \\ Keywords: EDO-S101; brain tumors; CNS Iymphoma; glioblastoma multiforme; metastatic breast cancer of the brain \\ Received: November 27, $2017 \quad$ Accepted: May 24, $2018 \quad$ Published: June 15, 2018 \\ Copyright: Qiu et al. This is an open-access article distributed under the terms of the Creative Commons Attribution License 3.0 \\ (CC BY 3.0), which permits unrestricted use, distribution, and reproduction in any medium, provided the original author and source \\ are credited.
}

\section{ABSTRACT}

There is a clear unmet need for novel therapeutic agents for management of primary and secondary brain tumors. Novel therapeutic agents with excellent central nervous system (CNS) penetration and therapeutic activity are urgently needed. EDO-S101 is a novel alkylating and histone deacetylase inhibiting agent created by covalent fusion of bendamustine and vorinostat.

We used murine models to perform CNS pharmacokinetic analysis and preclinical therapeutic evaluation of EDO-S101 for CNS Iymphoma, metastatic triple-negative breast cancer of the brain, and glioblastoma multiforme. EDO-S101 has excellent CNS penetration of $13.8 \%$ and $16.5 \%$ by intravenous infusion and bolus administration respectively. It shows promising therapeutic activity against CNS lymphoma, metastatic triple-negative breast cancer of the brain, and glioblastoma multiforme with significant prolongation of survival compared to no-treatment controls. Therapeutic activity was higher with IV infusion compared to IV bolus. It should be evaluated further for therapeutic use in brain tumors.

\section{INTRODUCTION}

Management of primary and secondary brain tumors remains a challenge due to lack of efficacious therapeutic agents with adequate central nervous system (CNS) penetration. Most brain tumors remain incurable with grim prognosis. There is an unmet need for novel therapeutic agents for CNS lymphoma (CNSL), metastatic breast cancer of the brain (MBCB), and glioblastoma multiforme (GBM).

Primary CNSL (PCNSL) is an aggressive diffuse large B-cell lymphoma (DLBCL) mostly of activated B-cell phenotype, which is confined to the CNS [1-4] with an incidence rate of 0.47 per 100,000 person-years [5]. It is an aggressive brain tumor with an average survival time of 1.5 to 3.3 months in untreated patients $[6,7]$. The standard current treatment of PCNSL includes induction with high- dose methotrexate-based chemoimmunotherapy followed by consolidation with whole brain radiation, intensive chemotherapy, or high-dose chemotherapy followed by autologous stem cell transplantation [2-4]. These treatments are rather toxic and are not well tolerated, especially by elderly patients in whom the incidence of PCNSL has been rising [2-4]. Once it has relapsed, the prognosis is usually very poor due to limited options for efficacious treatments. Although the survival has improved from a median survival of 12 months 50 years ago to $40 \%$ long-term survival at present, it is projected to plateau soon with currently available therapeutic agents [8]. As such, PCNSL remains a devastating brain tumor for which novel therapies are critically needed.

$\mathrm{MBCB}$ is a very serious event in the natural history of breast cancer and is associated with very poor 
prognosis $[9,10]$. The incidence of clinically evident brain metastases among women with stage IV breast cancer is $10 \%$ to $16 \%$ [10]. The true incidence is likely higher as brain metastases are found in $30 \%$ of patients at autopsy [10]. Therapy for systemic breast cancer has advanced with development of novel targeted therapeutic agents, resulting in improvement in survival of breast cancer patients. However, the same cannot be said for MBCB. Moreover, there has been an increase in the incidence of $\mathrm{MBCB}$ as breast cancer patients are surviving longer [10]. Triple-negative breast cancer is the most aggressive subtype of breast cancer with a high risk for brain metastases [9]. Currently, there is no effective systemic therapy for MBCB [10-12]. Additionally, therapeutic options after failure of radiation therapy are extremely limited [10]. Therapeutic agents with adequate CNS penetration and activity against breast cancer are needed.

The most common and aggressive primary brain tumor is GBM (World Health Organization grade IV astrocytoma) with an incidence rate of 3.19 per 100,000 person-years $[13,14]$. In spite of intensive research over the last few decades, impactful progress with improvement in survival has been elusive with the most recent data indicating median survival of 14.6 months $[13,14]$. The current therapeutic approach consists of maximal surgical total resection followed by postoperative radiation therapy with concurrent and adjuvant alkylating chemotherapy using temozolomide [14-17]. Response to temozolomide is strongly predicted by methylation status of the promoter of O [6]-methylguanine-DNA methyltransferase (MGMT), a DNA repair enzyme for alkylator-induced DNA damage $[14,18]$. Temozolomide does not work well in patients whose tumors are characterized by a lack of MGMT promoter methylation $[14,18]$. Bevacizumab, an antivascular endothelial growth factor therapy, has not shown great benefit for newly-diagnosed $[19,20]$ or recurrent $[21,22]$ GBM.

EDO-S101 is a novel first-in-class fusion molecule of an alkylator, bendamustine, and a histone-deacetylase (HDAC) inhibitor, vorinostat, also known as SAHA [23, 24] (Figure 1). It embodies a novel concept of synergism between alkylation and HDAC inhibition and introduces the alkylating-HDAC inhibition fusion principle [2328]. Mechanistically, it is a fully bifunctional molecule with retention of alkylating and pan-HDAC inhibitory activities [23, 24]. It induces DNA crosslinking by alkylation, which is 5 times more potent than melphalan and 10 times more potent than bendamustine [23, 24]. Its pan-HDAC inhibitory activity is as strong as vorinostat and is seen at nanomolar concentrations [23, 24]. HDAC inhibitory activity promotes open chromatin conformation via increased acetylation of histones allowing increased accessibility for DNA double strandbreaking by alkylating activity with resultant apoptosis of cancer cells [23, 25-28]. It has been shown to induce apoptosis via intrinsic pathway and cell cycle arrest at G2M phase [23]. It is delivered as a single intact molecule to the cancer cells ensuring generation of alkylation and HDAC inhibition simultaneously in the same location and creating therapeutic synergistic effect [23]. Concomitant exposure to both agents has been shown to be important to achieve maximal therapeutic effect [23, 29]. Pharmacodynamically, concomitant exposure to alkylation and HDAC inhibition cannot be expected to take place simultaneously in a reliable manner if the two agents are administered separately.

The unique mechanistic attributes of EDO-S101 make it a very potent antineoplastic agent, as evidenced by broad-spectrum cytotoxicity against multiple cancer types when tested against the NCI-60 panel of cancer cell

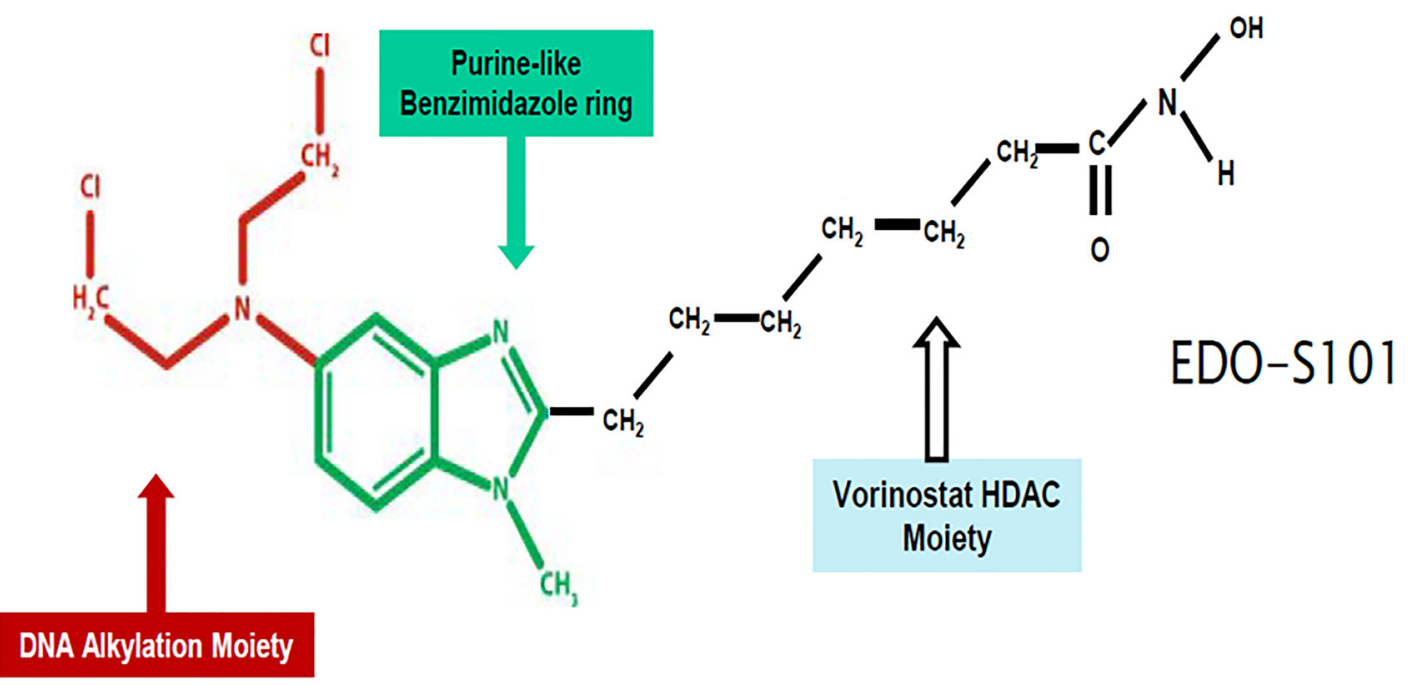

Figure 1: EDO-S101 structure. 
lines [23]. EDO-S101 is about 33 times more potent than bendamustine against NCI-60 cell lines with a median $\mathrm{IC}_{50}$ of $2.2 \mathrm{uM}$ versus $72.0 \mathrm{uM}$ for bendamustine [23]. Among the cell lines on NCI-60 panels with excellent response to EDO-S101 are GBM and breast cancer cell lines [23]. EDO-S101 has also been tested against hematologic cancer cell lines including lymphoma and leukemia, showing potent cytotoxicity with $\mathrm{IC}_{50}$ ranging from $0.4 \mathrm{uM}$ to $5.0 \mathrm{uM}$ [23]. It is currently undergoing a phase 1 clinical trial for relapsed and refractory hematologic malignancies (ClinicalTrials.gov identifier- NCT02576496).

\section{RESULTS}

\section{CNS pharmacokinetics of EDO-S101}

Excellent CNS penetration with adequate therapeutic CNS concentration is an essential prerequisite of antineoplastic agents for the treatment of brain tumors. We performed a CNS pharmacokinetic analysis on EDO-S101 administered by 2 schedules, IV bolus 40 $\mathrm{mg} / \mathrm{kg}$ in SD rats and CIVI $30 \mathrm{mg} / \mathrm{kg}$ over one hour in C57BL/6J mice. Pharmacokinetic findings are presented in Figure 2 and Figure 3. EDO-S101 was cleared quickly from both the blood and the brain with a short half-life. It can cross the blood-brain barrier with CNS penetration of $16.5 \%$ and $13.8 \%$ for IV bolus and CIVI administrations, respectively. The maximum concentration in the brain achieved by both administration methods was higher than the median $\mathrm{IC}_{50}$ value of $2.2 \mathrm{uM}$ for NCI-60 cancer cell lines and $\mathrm{IC}_{50}$ values of $0.4 \mathrm{uM}$ to $5.0 \mathrm{uM}$ for hematologic malignancy cancer cell lines [23], indicating that EDO-S101 achieves adequate therapeutic concentration in the brain. The CNS pharmacokinetic findings indicate that EDO-S101 is a good candidate for assessment of therapeutic activity against brain tumors.

\section{Therapeutic activity of EDO-S101 against CNSL in OCI-LY10 murine model}

PCNSL is a DLBCL confined to the CNS [2, 4]. Most of the patients with CNSL cannot be cured with the current treatments, which are rather toxic and not well tolerated $[2,4]$. As such, there is a need for novel therapeutic agents. Bendamustine has shown antilymphoma activity and is currently used as a chemotherapeutic agent for various types of lymphoma including indolent non-Hodgkin lymphoma, mantle cell lymphoma, DLBCL, and Hodgkin lymphoma [30]. Vorinostat has also shown antilymphoma activity, especially against cutaneous $\mathrm{T}$ cell lymphoma [31-33]. EDO-S101 has shown potent cytotoxic activity against DLBCL [23]. In a study on diffuse large B cell lymphoma cell lines, the concomitant treatment with bendamustine and vorinostat showed enhanced histone acetylation and double strand DNA breaks resulting in an additive to synergistic cytotoxic effect in both ABC- and GCBtype DLBCL cells independent of p53 mutation status [29]. However, improved cytotoxicity is not seen when lymphoma cells are treated sequentially [29].

Therapeutic activity of EDO-S101 against CNSL was tested in a murine model created by intracerebral implantation in athymic mice of OCI-LY10 DLBCL cells, which are the same subtype of DLBCL as PCNSL (activated B-cell subtype). EDO-S101 was administered by repeat IV bolus and repeat CIVI schedules.

Both treatment schedules showed significant therapeutic activity with suppression of tumor growth and prolongation of survival compared to the dimethyl sulfoxide (DMSO) control group (Figure 4). Median survival was 54 days in the EDO-S101 by repeat IV bolus treatment group vs 46 days in the DMSO control group, whereas it was 60 days in the repeat CIVI treatment group
A

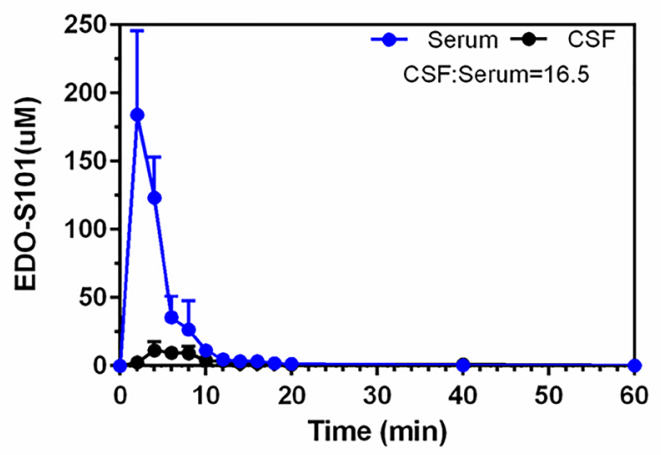

B

\begin{tabular}{|l|c|c|}
\hline \multirow{2}{*}{ PK Parameters } & \multicolumn{2}{|c|}{ PK values (Mean \pm SD) } \\
\cline { 2 - 3 } Cmax (uM) & Blood & Brain \\
\hline$T m a x(m i n)$ & $184.0 \pm 61.8$ & $11.2 \pm 6.5$ \\
\hline$T 1 / 2$ (min) & $2.33 \pm 0.82$ & $5.67 \pm 1.97$ \\
\hline AUC(0-12) (uM.hr) & $5.6 \pm 1.07$ & $8.8 \pm 1.43$ \\
\hline AUC ratio (Brain:Blood) & $824.3 \pm 110.8$ & $136.2 \pm 74.7$ \\
\hline
\end{tabular}

Figure 2: CNS pharmacokinetic analysis of EDO-S101 administered by IV bolus in SD rats (N=6). EDO-S101 $40 \mathrm{mg} /$ $\mathrm{kg}$ was given IV bolus followed by collection of microdialysates at regular time intervals over 60 minutes from the blood and the brain via microdialysis catheters placed in a carotid artery and a cerebral ventricle. EDO-S101 levels were determined by capillary electrophoresis. (A) Time concentration curves of EDO-S101 in the blood and the brain are shown. (B) Pharmacokinetic parameters of EDO-S101 in the blood and the brain are shown. CNS penetration of EDO-S101 is $\sim 16.5$ as calculated by the AUC ratio of brain and blood. 
vs 49 days in the control group. EDO-S101 treatment appeared to be better tolerated with the CIVI schedule compared to the IV bolus schedule based on observation of clinical toxicities including changes in weight, mobility, and feeding.

\section{Therapeutic activity of EDO-S101 against triple-negative MBCB in MB-468 murine model}

MBCB carries a very poor prognosis as no efficacious treatments are currently available [10]. Novel therapeutic agents that produced substantial improvement in non-CNS disease unfortunately do not cross the bloodbrain barrier well and have not had any major impact on prognosis of CNS disease [10]. EDO-S101 showed significant cytotoxicity against breast cancer cell lines in the NCI-60 panel (MCF7, BT-549, MDA-MB 231, T47D, MDA-MB-468, and HS578T) [23]. BT-549, MDA-MB 231, MDA-MB-468, and HS578T are triple negative breast cancer cell lines on the panel and showed significant sensitivity to EDO-S101 [23]. Among subtypes of breast cancer, triple-negative breast cancer is the most aggressive and has predilection for metastasizing to the brain [9].

Therapeutic activity of EDO-S101 against triplenegative $\mathrm{MBCB}$ was tested in a murine model created by intracerebral implantation of triple-negative MDAMB-468 breast cancer cells in athymic mice. Both repeat IV bolus and repeat CIVI treatment schedules showed significant therapeutic activity with suppression of tumor growth and prolongation of survival compared to DMSO control group (Figure 5). Median survival was 52 days in the EDO-S101 by repeat IV bolus treatment group vs 46 days in the DMSO control group, whereas it was 42 days in the repeat CIVI treatment group vs 27 days in the control group. EDO-S101 treatment appeared to be better tolerated with the CIVI schedule compared to the IV bolus schedule based on clinical observation.

\section{Therapeutic activity of EDO-S101 against GBM in a patient-derived xenograft model}

GBM is the most common and aggressive primary brain tumor [14]. In spite of intensive research efforts, GBM remains incurable $[13,14]$. Novel efficacious therapeutic agents are needed to fill this unmet therapeutic void. The standard chemotherapeutic agent for GBM is an alkylating agent, temozolomide [34, 35]. HDAC inhibition with vorinostat has shown therapeutic activity against GBM in a clinical trial [36]. As such, EDO-S101 with bifunctional activity is a promising therapeutic agent for GBM from a mechanistic standpoint. EDO-S101 showed notable in vitro cytotoxicity against GBM cell lines on NCI-60 panel (SF-268, SF-295, SF-539, SNB-19, SNB-75, and U-251), showing activity against GBM cell lines with both methylated and unmethylated MGMT promotor [23].

Therapeutic activity of EDO-S101 against GBM was tested in a GBM12 patient-derived xenograft model. Biologically, GBM12 cells show methylation of the promoter of MGMT and sensitivity to temozolomide [37]. Both repeat IV bolus and repeat CIVI treatment schedules showed significant therapeutic activity with suppression of tumor growth and prolongation of survival compared to the DMSO control group (Figure 6). Median survival was 62 days in the EDO-S101 by repeat IV bolus treatment group vs 52 days in the DMSO control group, whereas it was 80 days in the repeat CIVI treatment group vs 63 days in the control group. Similar to CNSL and MBCB experiments, EDO-S101 treatment appeared to be better tolerated with the CIVI schedule compared to IV bolus schedule based on observation of clinical toxicities.
A

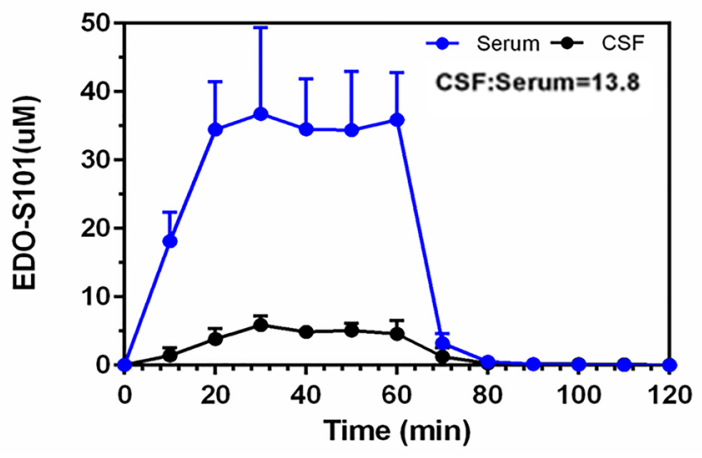

B

\begin{tabular}{|l|c|c|}
\hline \multirow{2}{*}{\multicolumn{2}{|c|}{ PK Parameters }} & \multicolumn{2}{c|}{ PK values (Mean \pm SD) } \\
\cline { 2 - 3 } Cmax (UM) & $36.31 \pm 11.8$ & Brain \\
\hline Tmax (min) & $31.20 \pm 10.84$ & $30.37 \pm 8.37$ \\
\hline$T 1 / 2$ (min) & $36.81 \pm 5.79$ & $37.63 \pm 6.07$ \\
\hline AUC(0-12) (uM.hr) & $1980.26 \pm 208.4$ & $273.8 \pm 67.7$ \\
\hline AUC ratio (Brain:Blood) & \multicolumn{2}{|c|}{$13.8 \pm 3.69$} \\
\hline
\end{tabular}

Figure 3: CNS pharmacokinetic analysis of EDO-S101 administered by one hour IV infusion in C57BL/6J (N=6). EDO-S101 $30 \mathrm{mg} / \mathrm{kg}$ was given by IV infusion over one hour followed by collection of microdialysates at regular time intervals over 120 minutes from the blood and the brain via microdialysis catheters placed in a carotid artery and a cerebral ventricle. EDO-S101 levels were determined by capillary electrophoresis. (A) Time concentration curves of EDO-S101 in the blood and the brain are shown. (B) Pharmacokinetic parameters of EDO-S101 in the blood and the brain are shown. CNS penetration of EDO-S101 is $\sim 13.8$ as calculated by the AUC ratio of brain and blood. 


\section{DISCUSSION}

Our results indicate that EDO-S101 is a novel CNSpenetrating antineoplastic agent with impressive preclinical therapeutic activity against 3 aggressive brain tumors (CNSL, MBCB, and GBM). Our CNS pharmacokinetic analysis showed excellent CNS penetration, which enables it to achieve adequate therapeutic CNS concentration. Its maximum concentration in the blood and brain are substantially higher than its median $\mathrm{IC}_{50}$ value for NCI60 cell lines and $\mathrm{IC}_{50}$ values for hematologic cancer cell lines. As such, it likely has broad-spectrum therapeutic activity against primary and secondary brain tumors. Bifunctional mechanistic activity of EDO-S101 with synergism between simultaneous alkylation and HDAC

A

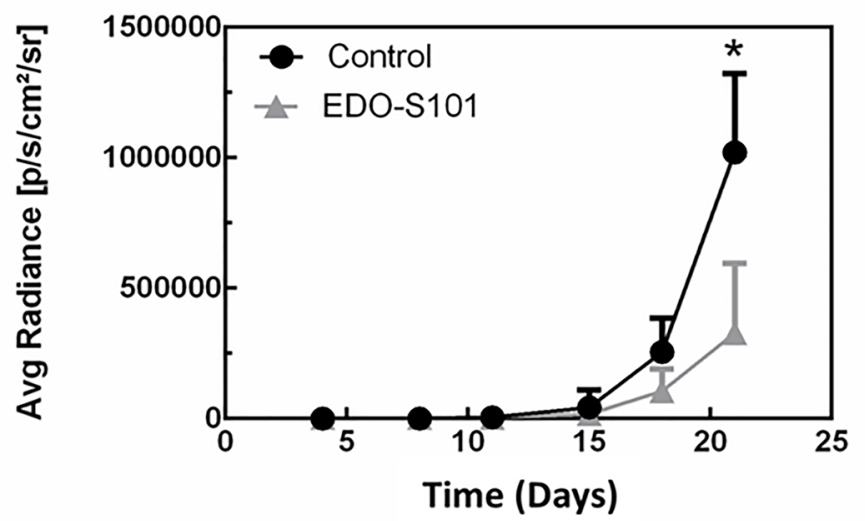

C

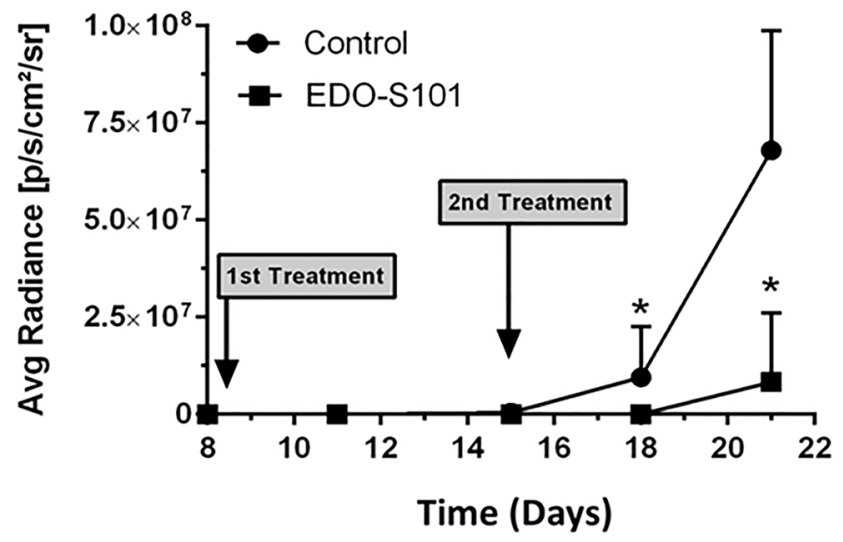

inhibition taking place in the cancer cells may explain its unique therapeutic activity.

Currently, there is an unmet therapeutic need for the 3 brain tumors for which we tested EDO-S101 in preclinical models. Primary and secondary CNSLs remain mostly incurable with currently available treatments, which are rather toxic and not well tolerated [2-4]. In a small retrospective study in patients with recurrent PCNSL refractory to high-dose methotrexate, bendamustine was shown to have modest single-agent activity with manageable toxicity [38]. EDO-S101 should be evaluated further in CNSL.

Although great strides have been made over the last few decades leading to improvement in the prognosis of systemic breast cancer, very little improvement has been

B

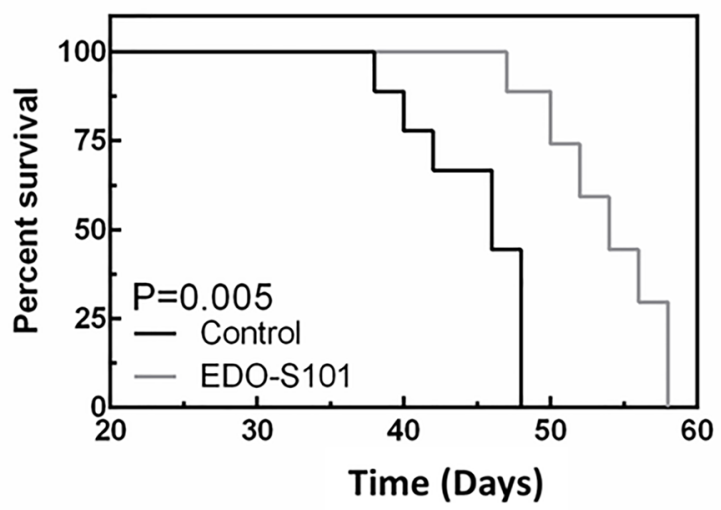

D

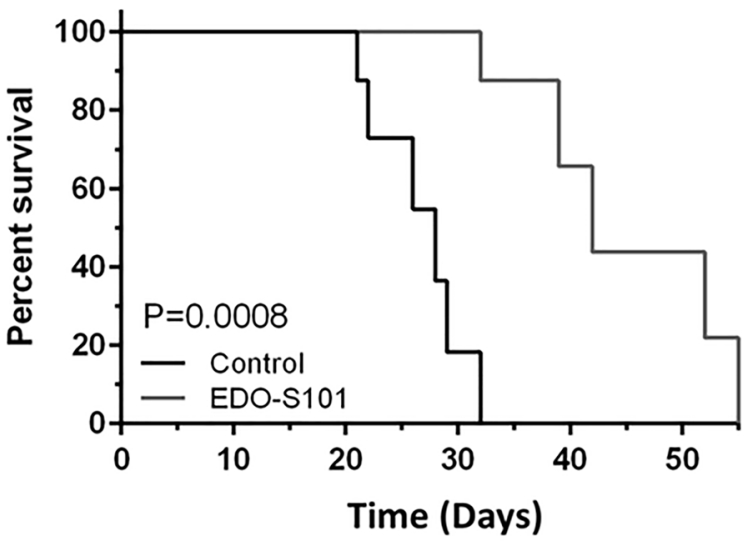

Figure 4: EDO-S101 has significant therapeutic activity against CNS lymphoma. Therapeutic activity of EDO-S101 against CNS lymphoma was tested in OCI-LY10 murine model. $5 \times 10^{5}$ luciferase-transfected OCI-LY10 lymphoma cells were intracerebrally injected in L periventricular area in athymic mice. Therapeutic activity was assessed by impact on tumor growth as reflected by bioluminescence activity (A \& C) and survival analysis (B \& D). Mice in control group received DMSO on the similar treatment schedule as EDO-S101 treatment group. N=10 in treatment and control groups. (A \& B) Repeat IV bolus administration of EDO-S101. The treatments were given on days 4, 11, and 18 post tumor implantation. (C \& D) Repeat continuous IV infusion of EDO-S101. The treatments were given on days 8 and 15 post tumor implantation by IV infusion over one hour via an infusion pump. EDO-S101 treatment resulted in suppression of tumor growth which translated into significant prolongation of survival $(\mathrm{P}<0.05)$. 
A

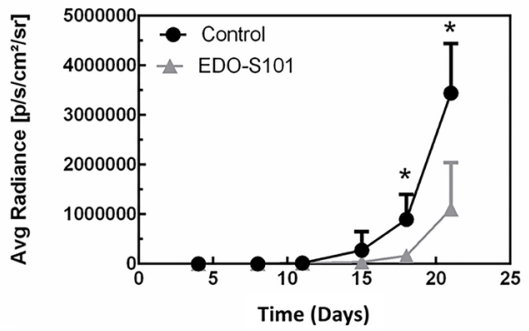

C

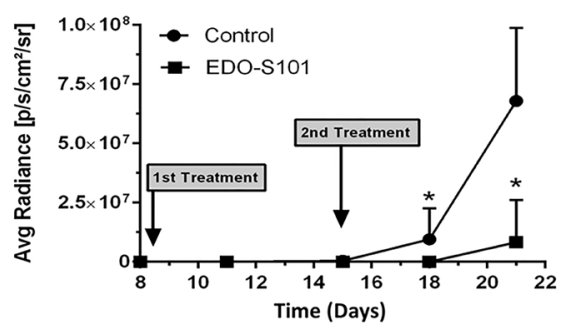

B

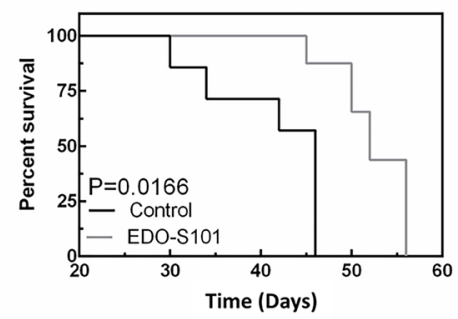

D

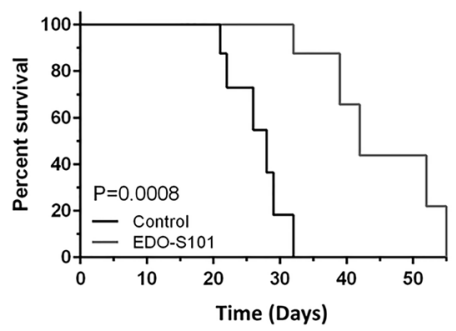

Figure 5: EDO-S101 has significant therapeutic activity against metastatic breast cancer of the brain. Therapeutic activity of EDO-S101 against metastatic breast cancer of the brain was tested in MB-468 murine model. $5 \times 10^{5}$ luciferase-transfected MB-468 triple negative breast cancer cells were intracerebrally injected in L periventricular area in athymic mice. Therapeutic activity was assessed by impact on tumor growth as reflected by bioluminescence activity (A \& C) and survival analysis (B \& D). Mice in control group received DMSO on the similar treatment schedule as EDO-S101 treatment group. $\mathrm{N}=10$ in treatment and control groups. (A \& B) Repeat IV bolus administration of EDO-S101. The treatments were given on days 4, 11, and 18 post tumor implantation. (C \& D) Repeat continuous IV infusion of EDO-S101. The treatments were given on days 8 and 15 post tumor implantation by IV infusion over one hour via an infusion pump. EDO-S101 treatment resulted in suppression of tumor growth which translated into significant prolongation of survival $(\mathrm{P}<0.05)$.

A

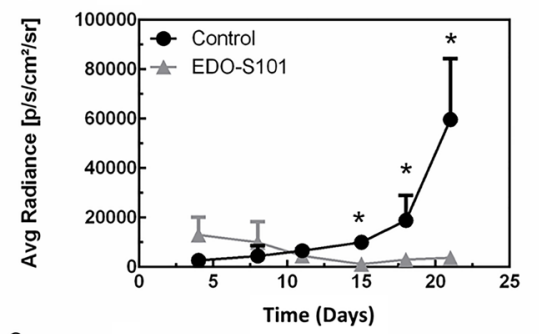

C

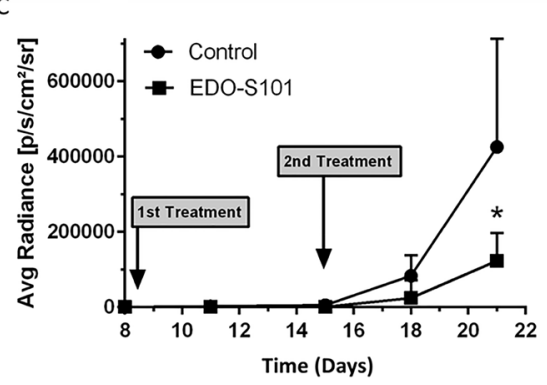

B

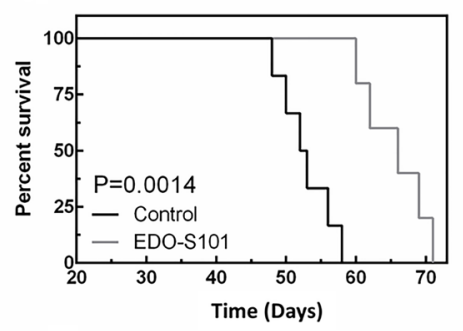

D

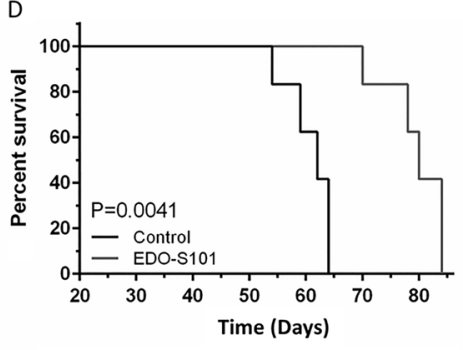

Figure 6: EDO-S101 has significant therapeutic activity against glioblastoma multiforme. Therapeutic activity of EDO-S101 against glioblastoma multiforme was tested in GBM12 patient derived xenograft model. 5 x $10^{5}$ luciferase-transfected GBM12 cells were intracerebrally injected in L periventricular area in athymic mice. Therapeutic activity was assessed by impact on tumor growth as reflected by bioluminescence activity (A \& C) and survival analysis (B \& D). Mice in control group received DMSO on the similar treatment schedule as EDO-S101 treatment group. $\mathrm{N}=10$ in treatment and control groups. (A \& B) Repeat IV bolus administration of EDO-S101. The treatments were given on days 4, 11, and 18 post tumor implantation. (C \& D) Repeat continuous IV infusion of EDO-S101. The treatments were given on days 8 and 15 post tumor implantation by IV infusion over one hour via an infusion pump. EDO-S101 treatment resulted in suppression of tumor growth which translated into significant prolongation of survival $(\mathrm{P}<0.05)$. 
seen for MBCB. This grim situation exists mainly because the novel therapeutic agents developed over the last few decades do not adequately penetrate the brain. Excellent CNS penetration of EDO-S101 combined with our in vivo results and excellent cytotoxicity against NCI-60 breast cancer cell lines are encouraging.

The preclinical therapeutic findings of EDO-S101 against GBM are quite exciting. Alkylation (temozolomide) $[34,35]$ and HDAC inhibition (vorinostat) [36] have been associated with therapeutic activity in GBM. However, a recent phase I/II trial of vorinostat combined with temozolomide and radiation therapy for newly diagnosed glioblastoma did not demonstrate significant activity [39]. We have to note that vorinostat and temozolomide administered separately cannot be considered as equivalent to EDO-S101, which is a fusion drug consisting of bendamustine and vorinostat. Moreover, EDO-S101 has been shown to have activity against temozolomide-sensitive as well as resistant GBM cell lines [23]. As such, the clinical trial findings cannot be used to predict therapeutic efficacy of EDO-S101. As EDO-S101 has a unique synergistic bifunctionality of alkylation and HDAC inhibition, it represents a new therapeutic approach for GBM and should be explored further in other preclinical GBM models representing various genetic and molecular subsets with the ultimate aim of translating into a phase 1 clinical trial.

In this study, we tested 2 treatment schedules, repeat IV bolus and repeat 1-hour CIVI. Both administration schedules were associated with therapeutic activity. The total dose of EDO-S101 administered was $60 \mathrm{mg} / \mathrm{kg}$ and $180 \mathrm{mg} / \mathrm{kg}$ for CIVI and IV bolus schedules, respectively. In spite of this degree of difference in dose intensity, considerable therapeutic activity was still observed with the CIVI schedule. The CIVI schedule appears to be better tolerated based on our observation of clinical toxicity in animals, indicating that there is still a lot of room for dose intensification for this treatment schedule. As such, the CIVI schedule appears to be more suitable for further evaluation.

In conclusion, EDO-S101 holds promise for the treatment of primary and secondary brain tumors and should be further evaluated in clinical trials.

\section{MATERIALS AND METHODS}

\section{CNS pharmacokinetic analysis}

CNS pharmacokinetic analysis was performed in murine models to determine the CNS penetration of EDO-S101. EDO-S101 was administered by singledose intravenous (IV) bolus $(40 \mathrm{mg} / \mathrm{kg}$ ) in SD rats or continuous IV infusion (CIVI; $30 \mathrm{mg} / \mathrm{kg}$ ) for 1 hour via an infusion pump in $\mathrm{C} 57 \mathrm{BL} / 6 \mathrm{~J}$ mice. Microdialysates were collected at regular time intervals from the brain or blood via microdialysis catheters in a ventricle of the brain and a carotid artery. Concentration of the drug in the microdialysate samples is determined by capillary electrophoresis with ultraviolet detection at $280 \mathrm{nM}$. Details of microdialysis and capillary electrophoresis were previously published [40]. Time-concentration graphs were constructed and CNS pharmacokinetic parameters were calculated. CNS penetration of the drug is calculated as the percent area under the curve ratio of brain and blood.

\section{Preclinical brain tumor models}

Details of intracerebral implantation of tumor cells were previously published [41]. The CNSL model was created by intracerebral injection of luciferase-transfected OCI-LY10 DLBCL cells into the left periventricular area of the brain in athymic mice. The cells were provided by Arthur L. Shaffer, III, National Cancer Institute of Health, Bethesda, Maryland. The glioblastoma patient-derived xenograft model was created by intracerebral injection of luciferase-transfected primary GBM cells (GBM12) into left periventricular area of the brain in athymic mice. The cells were provided by Dr. Jan Sakaria, Mayo Clinic, Rochester, Minnesota [37]. The MBCB model was created by intracerebral injection of luciferase-transfected MB-468 triple-negative breast cancer cells into left periventricular area of the brain in athymic mice. The cells were purchased from ATCC (Manassas, Virginia).

\section{Treatment with EDO-S101}

In the IV bolus group, $60 \mathrm{mg} / \mathrm{kg}$ of EDO-S101 was administered by repeat IV bolus via tail vein injection on days 4,11 , and 18 after tumor implantation. In the CIVI group, $30 \mathrm{mg} / \mathrm{kg}$ of EDO-S101 was administered by repeat CIVI via an infusion port over 1 hour on days 8 and 15 after tumor implantation.

\section{Bioluminescence imaging}

Bioluminescence imaging was used to study the impact of treatment on intracerebral tumor growth in real time. The detailed methodology has been previously published [41].

\section{Statistics}

Analysis of variance was used to determine statistical significance of the differences between experimental groups. Survival analysis was performed by Kaplan-Meier method using limb paralysis as the end point. Kaplan-Meier survival curves were generated using Prism4 software (GraphPad Software, LaJolla, CA), and the statistical difference between curves was derived with a log-rank test. $P<.05$ was considered significant. 


\section{Abbreviations}

Central nervous system (CNS)

Metastatic breast cancer of the brain (MBCB)

Glioblastoma multiforme (GBM)

Diffuse large B-cell lymphoma (DLBCL)

$\mathrm{O}$ [6]-methylguanine-DNA methyltransferase (MGMT)

Histone-deacetylase (HDAC)

Continuous IV infusion (CIVI)

Dimethyl sulfoxide (DMSO).

\section{Author contributions}

Yushi Qiu performs the experiments, analyzes the data and writes the manuscript. the data.

Zhimin Li performs the experiments and analyzes

John A Copland provides reagents and analyzes the data.

Thomas Mehrling provides reagents.

Han W Tun provides reagents, performs the experiments, analyzes the data and writes the manuscript.

\section{CONFLICTS OF INTEREST}

Han W. Tun received a research grant from Mundipharma-EDO GmbH. Thomas Mehrling is an employee of Mundipharma-EDO GmbH.

\section{FUNDING}

Han W. Tun is supported by James C, Sara K. Kennedy award and a research grant from Mundipharma. John A. Copland is supported by NCI RO1CA136665 and Betty G. Castigliano Fund in Cancer Research Honoring S. Gordon Castigliano.

\section{REFERENCES}

1. Campo E, Swerdlow SH, Harris NL, Pileri S, Stein H, Jaffe ES. The 2008 WHO classification of lymphoid neoplasms and beyond: evolving concepts and practical applications. Blood. 2011; 117:5019-32. https://doi.org/10.1182/ blood-2011-01-293050.

2. Ferreri AJ. How I treat primary CNS lymphoma. Blood. 2011; 118:510-22. https://doi.org/10.1182/ blood-2011-03-321349.

3. Grommes C, DeAngelis LM. Primary CNS Lymphoma. J Clin Oncol. 2017; 35:2410-18. https://doi.org/10.1200/ JCO.2017.72.7602.

4. Rubenstein JL, Gupta NK, Mannis GN, Lamarre AK, Treseler P. How I treat CNS lymphomas. Blood. 2013; 122:2318-30. https://doi.org/10.1182/ blood-2013-06-453084.
5. Villano JL, Koshy M, Shaikh H, Dolecek TA, McCarthy BJ. Age, gender, and racial differences in incidence and survival in primary CNS lymphoma. Br J Cancer. 2011; 105:1414-18. https://doi.org/10.1038/bjc.2011.357.

6. Schabet M. Epidemiology of primary CNS lymphoma. J Neurooncol. 1999; 43:199-201. https://doi. org/10.1023/A:1006290032052.

7. Zimmerman HM. Malignant lymphomas of the nervous system. Acta Neuropathol Suppl. 1975; 6:69-74.

8. Ponzoni M, Issa S, Batchelor TT, Rubenstein JL. Beyond high-dose methotrexate and brain radiotherapy: novel targets and agents for primary CNS lymphoma. Ann Oncol. 2014; 25:316-22. https://doi.org/10.1093/annonc/mdt385.

9. Kennecke H, Yerushalmi R, Woods R, Cheang MC, Voduc D, Speers CH, Nielsen TO, Gelmon K. Metastatic behavior of breast cancer subtypes. J Clin Oncol. 2010; 28:3271-77. https://doi.org/10.1200/JCO.2009.25.9820.

10. Lin NU, Bellon JR, Winer EP. CNS metastases in breast cancer. J Clin Oncol. 2004; 22:3608-17. https://doi. org/10.1200/JCO.2004.01.175.

11. Freedman RA, Anders CK. Treatment of Breast Cancer Brain Metastases. Curr Breast Cancer Rep. 2012; 4:1-9. https://doi.org/10.1007/s12609-011-0061-5.

12. Ogawa K, Yoshii Y, Nishimaki T, Tamaki N, Miyaguni T, Tsuchida Y, Kamada Y, Toita T, Kakinohana Y, Tamaki W, Iraha S, Adachi G, Hyodo A, Murayama S. Treatment and prognosis of brain metastases from breast cancer. J Neurooncol. 2008; 86:231-38. https://doi.org/10.1007/ s11060-007-9469-1.

13. Glioblastoma Information (American Brain Tumor Association). 2016. https:/www.abta.org/tumor_types/ glioblastoma-gbm/.

14. Alexander BM, Cloughesy TF. Adult Glioblastoma. J Clin Oncol. 2017; 35:2402-09. https://doi.org/10.1200/ JCO.2017.73.0119.

15. Stupp R, van den Bent MJ, Hegi ME. Optimal role of temozolomide in the treatment of malignant gliomas. Curr Neurol Neurosci Rep. 2005; 5:198-206. https://doi. org/10.1007/s11910-005-0047-7.

16. Stupp R, Mason WP, van den Bent MJ, Weller M, Fisher B, Taphoorn MJ, Belanger K, Brandes AA, Marosi C, Bogdahn U, Curschmann J, Janzer RC, Ludwin SK, et al, and European Organisation for Research and Treatment of Cancer Brain Tumor and Radiotherapy Groups, and National Cancer Institute of Canada Clinical Trials Group. Radiotherapy plus concomitant and adjuvant temozolomide for glioblastoma. N Engl J Med. 2005; 352:987-96. https:// doi.org/10.1056/NEJMoa043330.

17. Stupp R, Hegi ME, Mason WP, van den Bent MJ, Taphoorn MJ, Janzer RC, Ludwin SK, Allgeier A, Fisher B, Belanger K, Hau P, Brandes AA, Gijtenbeek J, et al, and European Organisation for Research and Treatment of Cancer Brain Tumour and Radiation Oncology Groups, and National Cancer Institute of Canada Clinical Trials Group. Effects of 
radiotherapy with concomitant and adjuvant temozolomide versus radiotherapy alone on survival in glioblastoma in a randomised phase III study: 5-year analysis of the EORTCNCIC trial. Lancet Oncol. 2009; 10:459-66. https://doi. org/10.1016/S1470-2045(09)70025-7.

18. Thon N, Kreth S, Kreth FW. Personalized treatment strategies in glioblastoma: MGMT promoter methylation status. Onco Targets Ther. 2013; 6:1363-72. https://doi. org/10.2147/OTT.S50208.

19. Chinot OL, Wick W, Mason W, Henriksson R, Saran F, Nishikawa R, Carpentier AF, Hoang-Xuan K, Kavan P, Cernea D, Brandes AA, Hilton M, Abrey L, Cloughesy T. Bevacizumab plus radiotherapy-temozolomide for newly diagnosed glioblastoma. N Engl J Med. 2014; 370:709-22. https://doi.org/10.1056/NEJMoa1308345.

20. Gilbert MR, Dignam JJ, Armstrong TS, Wefel JS, Blumenthal DT, Vogelbaum MA, Colman H, Chakravarti A, Pugh S, Won M, Jeraj R, Brown PD, Jaeckle KA, et al. A randomized trial of bevacizumab for newly diagnosed glioblastoma. N Engl J Med. 2014; 370:699-708. https:// doi.org/10.1056/NEJMoa1308573.

21. Friedman HS, Prados MD, Wen PY, Mikkelsen T, Schiff D, Abrey LE, Yung WK, Paleologos N, Nicholas MK, Jensen R, Vredenburgh J, Huang J, Zheng M, Cloughesy $\mathrm{T}$. Bevacizumab alone and in combination with irinotecan in recurrent glioblastoma. J Clin Oncol. 2009; 27:4733-40. https://doi.org/10.1200/JCO.2008.19.8721.

22. Taal W, Oosterkamp HM, Walenkamp AM, Dubbink HJ, Beerepoot LV, Hanse MC, Buter J, Honkoop AH, Boerman D, de Vos FY, Dinjens WN, Enting RH, Taphoorn MJ, et al. Single-agent bevacizumab or lomustine versus a combination of bevacizumab plus lomustine in patients with recurrent glioblastoma (BELOB trial): a randomised controlled phase 2 trial. Lancet Oncol. 2014; 15:943-53. https://doi.org/10.1016/S1470-2045(14)70314-6.

23. GmbH ME. Investigator's Brochure- EDO-S101. 2015; Edition 1.0.

24. Mehrling T, Chen Y. The Alkylating-HDAC inhibition fusion principle: taking chemotherapy to the next level with the first in class molecule EDO-S101. Anticancer Agents Med Chem. 2016; 16:20-28. https://doi.org/10.2174/1871 520615666150518092027.

25. Groselj B, Sharma NL, Hamdy FC, Kerr M, Kiltie AE. Histone deacetylase inhibitors as radiosensitisers: effects on DNA damage signalling and repair. Br J Cancer. 2013; 108:748-54. https://doi.org/10.1038/bjc.2013.21.

26. Kim HJ, Bae SC. Histone deacetylase inhibitors: molecular mechanisms of action and clinical trials as anti-cancer drugs. Am J Transl Res. 2011; 3:166-79.

27. Stiborová M, Eckschlager T, Poljaková J, Hraběta J, Adam V, Kizek R, Frei E. The synergistic effects of DNA-targeted chemotherapeutics and histone deacetylase inhibitors as therapeutic strategies for cancer treatment. Curr Med Chem. 2012; 19:4218-38. https://doi. org/10.2174/092986712802884286.
28. Thurn KT, Thomas S, Moore A, Munster PN. Rational therapeutic combinations with histone deacetylase inhibitors for the treatment of cancer. Future Oncol. 2011; 7:263-83. https://doi.org/10.2217/fon.11.2.

29. Fernández-Rodríguez C, Salar A, Navarro A, Gimeno E, Pairet S, Camacho L, Ferraro M, Serrano S, Besses C, Bellosillo B, Sanchez-Gonzalez B. Anti-tumor activity of the combination of bendamustine with vorinostat in diffuse large B-cell lymphoma cells. Leuk Lymphoma. 2016; 57:692-99. https://doi.org/10.3109/10428194.2015.10631 43.

30. Weidmann E, Kim SZ, Rost A, Schuppert H, Seipelt G, Hoelzer D, Mitrou PS. Bendamustine is effective in relapsed or refractory aggressive non-Hodgkin's lymphoma. Ann Oncol. 2002; 13:1285-9. https://doi.org/10.1093/ annonc/mdf189.

31. Kretzner L, Scuto A, Dino PM, Kowolik CM, Wu J, Ventura P, Jove R, Forman SJ, Yen Y, Kirschbaum MH. Combining histone deacetylase inhibitor vorinostat with aurora kinase inhibitors enhances lymphoma cell killing with repression of c-Myc, hTERT, and microRNA levels. Cancer Res. 2011; 71:3912-20. https://doi.org/10.1158/0008-5472. CAN-10-2259.

32. Kirschbaum MH, Goldman BH, Zain JM, Cook JR, Rimsza LM, Forman SJ, Fisher RI. A phase 2 study of vorinostat for treatment of relapsed or refractory Hodgkin lymphoma: Southwest Oncology Group Study S0517. Leuk Lymphoma. 2012; 53:259-62. https://doi.org/10.3109/10428194.2011.6 08448 .

33. Kirschbaum M, Frankel P, Popplewell L, Zain J, Delioukina M, Pullarkat V, Matsuoka D, Pulone B, Rotter AJ, EspinozaDelgado I, Nademanee A, Forman SJ, Gandara D, Newman E. Phase II study of vorinostat for treatment of relapsed or refractory indolent non-Hodgkin's lymphoma and mantle cell lymphoma. J Clin Oncol. 2011; 29:1198-203. https:// doi.org/10.1200/JCO.2010.32.1398.

34. Weller M, Cloughesy T, Perry JR, Wick W. Standards of care for treatment of recurrent glioblastoma--are we there yet? Neuro Oncol. 2013; 15:4-27. https://doi.org/10.1093/ neuonc/nos273.

35. Cloughesy TF, Cavenee WK, Mischel PS. Glioblastoma: from molecular pathology to targeted treatment. Annu Rev Pathol. 2014; 9:1-25. https://doi.org/10.1146/ annurev-pathol-011110-130324.

36. Galanis E, Jaeckle KA, Maurer MJ, Reid JM, Ames MM, Hardwick JS, Reilly JF, Loboda A, Nebozhyn M, Fantin VR, Richon VM, Scheithauer B, Giannini C, et al. Phase II trial of vorinostat in recurrent glioblastoma multiforme: a north central cancer treatment group study. J Clin Oncol. 2009; 27:2052-58. https://doi.org/10.1200/ JCO.2008.19.0694.

37. Carlson BL, Pokorny JL, Schroeder MA, Sarkaria JN. Establishment, maintenance and in vitro and in vivo applications of primary human glioblastoma multiforme (GBM) xenograft models for translational biology studies 
and drug discovery. Curr Protoc Pharmacol. 2011; Chapter 14:Unit 14.16. https://doi.org/10.1002/0471141755. ph1416s52.

38. Chamberlain MC. Salvage therapy with bendamustine for methotrexate refractory recurrent primary CNS lymphoma: a retrospective case series. J Neurooncol. 2014; 118:15562. https://doi.org/10.1007/s11060-014-1411-8.

39. Galanis E, Anderson SK, Miller CR, Sarkaria JN, Jaeckle $\mathrm{K}$, Buckner JC, Ligon KL, Ballman KV, Moore DF Jr, Nebozhyn M, Loboda A, Schiff D, Ahluwalia MS, et al. Phase I/II trial of vorinostat combined with temozolomide and radiation therapy for newly diagnosed glioblastoma: final results of alliance N0874/ABTC 02. Neuro-Oncology. 2018; 20:546-556. https://doi.org/10.1093/neuonc/nox161.
40. Li ZM, Shigeoka D, Caulfield TR, Kawachi T, Qiu YS, Kamon T, Arai M, Tun HW, Yoshimitsu T. An integrated approach to the discovery of potent agelastatin A analogues for brain tumors: chemical synthesis and biological, physicochemical and CNS pharmacokinetic analyses. MedChemComm. 2013; 4:1093-98. https://doi. org/10.1039/c3md00094j.

41. Li Z, Qiu Y, Personett D, Huang P, Edenfield B, Katz J, Babusis D, Tang Y, Shirely MA, Moghaddam MF, Copland JA, Tun HW. Pomalidomide shows significant therapeutic activity against CNS lymphoma with a major impact on the tumor microenvironment in murine models. PLoS One. 2013; 8:e71754. https://doi.org/10.1371/journal. pone. 0071754 . 